\title{
Research and Design of P2P-SIP System Network Structure Based on JXTA
}

\author{
WangFei, Dong $\mathbf{Y u}$ * \\ Henan Polytechnic Institute, Nanyang, Henan, China \\ *Corresponding Author.
}

\begin{abstract}
The network structure of P2P-SIP system based on JXTA is one of the core technologies in industrial intelligent Internet.JXTA is an open peer-to-peer platform, which provides basic application services for building P2P network. This paper chooses JXTA development platform to build P2P network, and then constructs SIP soft terminal on it. Firstly, it introduces the background knowledge, related research, P2P network technology, JXTA technology and SIP protocol. Then, the feasibility of $p 2 p$ sip system is analyzed, including the combination of P $2 P$ and sip, network structure and framework structure. Then the routing mechanism of JXTA is deeply studied, including distributed index of shared resources, limited range traversal, dynamic view of sink node and loose and consistent distributed hash table (DHT). At the same time, this paper proposes an improvement strategy to overcome the shortcomings of JXTA routing mechanism such as limited range traversal and loose consistent DHT, that is, introducing the chord ring of consistent hash algorithm in the aggregation network layer. Based on the indepth study of JXTA structured network and SIP protocol, a P2P SIP system is designed and implemented. The experimental results show that the system can maintain normal SIP communication without maintaining the central server.
\end{abstract}

Keywords: P2P SIP, JXTA, Network Structure, industrial intelligent Internet.

\section{Introduction}

In recent years, with the continuous development of IP (Internet Protocol) technology and the popularity of the Internet, people use more and more Internet based communication services, especially the demand and application of VoIP (voice over Internet Protocol) [1-2]. The rapid development of VoIP has completely subverted the traditional telecom business model. Its resource occupation has nothing to do with the cost, and the business and network operation are separated, which has a strong impact on the traditional telecom business model. The trend of IP phone system replacing the traditional PSTN (public switched telephone network) is irresistible. Therefore, VoIP technology has gradually become the object of current commercial consideration, and with the development of VoIP technology, the related communication services are also rapidly expanding. Network based video communication services, instant messaging services, user status rendering services also began to enter people's lives [3]. A variety of communication software came into being, providing a wealth of services, but also greatly promote the development of the Internet.

With the development of the Internet, P2P (peer-to-peer) technology has also developed rapidly in recent years, impacting many industries such as telecommunications and Internet. From the popular BitTorrent file download to the more and more popular video rebroadcasting, P2P technology has penetrated into the daily application of the Internet. Now the VoIP service launched by Skype also adopts P2P technology, and has achieved great success. $\mathrm{P} 2 \mathrm{P}$ network is a distributed peer-to-peer network without a specific central server, which is different from the traditional C / S (client / server) network architecture. The entities in P2P network are peer-to-peer, both client and server. All entities share resources. Compared with $\mathrm{C} / \mathrm{s}$ network architecture, P2P network architecture has the characteristics of decentralization, scalability, robustness, load balancing and so on. These advantages make P2P technology more and more attention [4-5]. With the continuous development and maturity of P2P technology, it

ISSN: 0010-8189

(C) CONVERTER 2021

Www.converter-magazine.info 
will bring great changes to the future Internet communication.

\section{P2P network technology}

\subsection{Definition of P2P network}

$\mathrm{P} 2 \mathrm{P}$ is the abbreviation of "peer-to-peer" in English. The English meaning of "peer" is "(status, ability, etc.) equal, colleague, partner". P2P can also be understood as "partner to partner", which is generally called "peer-to-peer network" in the computer field. Ian foster defines P2P computing technology as follows: P2P computing technology provides a decentralized, self-organized, all or most of the connections are symmetrical distributed environment for users and providers of various resources joining the Internet, and realizes the management of data information, storage space, computing power, functional components, and so on in a wide area Make full use of communication resources [6-7].

P2P network architecture breaks the traditional C / S network architecture. In P2P network, each node has the same status. It has the dual characteristics of client and server, and can act as a service user and a service provider at the same time. Due to the rapid development of P2P technology, the storage mode of the Internet will change from the current content centered mode to the content centered mode, change the current big website centered state of the Internet, return to the non centralized mode, and return the power to the users.

\subsection{P2P application field}

1. Content sharing. Content sharing is an early application of P2P. Napster, which appeared in the late 1990s, shared MP3 with friends and exchanged them. This event directly triggered the P2P technology revolution and made people realize the great development potential of P2P. Another kind of content sharing application is storage sharing. This kind of application uses the free disk space and memory in the whole P2P network to distribute the large-scale computing work to multiple computers, so as to reduce the burden of the server. OceanStore (mass storage system) is a typical application, which provides global distributed, continuous and stable data storage.

2. Distributed computing. Distributed computing is the use of P2P network idle CPU, disk space and memory to carry out large-scale operations. Its typical representative is SETI @ HOME (Search for Extra-Territorial Intelligence) system, which can integrate the processing and computing capabilities of computers connected to the Internet when they are idle to form a huge virtual computer.

3. Communication and cooperation. Communication and cooperation mainly include two types. One is Skype realtime communication software. Two or more users use text, voice or video to communicate with each other, and they can identify users without relying on devices. Because P2P network is decentralized, it can weaken or even get rid of the dependence on the central server [8-10]. The other is groove networks collaboration software, which is a software tool for collaboration on P2P network. It can build a secure enterprise level collaboration platform, provide interactive information communication on the supply and demand information chain, and help users to provide customer service and technical support.

4. Search engine. P2P technology can search resource information very well. This kind of search does not need to go through the central server, and is not limited by resource information format and host device. Infrasearch is a typical application of search engine technology, which is used to search resource information in P2P network.

\subsection{P2P network architecture}

1. Centralized directory P2P network architecture

Napster is the earliest centralized directory P2P network architecture, and it is also the most typical representative of the early P2P network. Napster saves the index and storage location information of all Napster users uploaded music files through a directory server. When a user needs a music file, it connects to Napster directory server first and searches it on the directory server. The directory server returns the user information or host information that contains the file, and then connects the requester directly to the owner for transmission. The centralized directory 
P2P network architecture is usually shown in Figure 1.

\section{Pure $\mathrm{P} 2 \mathrm{P}$ network architecture}

Pure P2P network architecture uses broadcast P2P model. Without the existence of directory index server, all queries are carried out through limited broadcast in the network. However, with the increasing number of network nodes and the expansion of network scale, the limited broadcast location of peer-to-peer nodes results in a sharp increase in network traffic, which leads to the failure of some low bandwidth nodes due to the overload of network resources. Gnutella is a typical representative of this kind of system, and it is also the most widely used pure P2P network architecture, which solves the problem of network structure centralization, and has good scalability and fault tolerance. The pure P2P network architecture is usually shown in Figure 2.

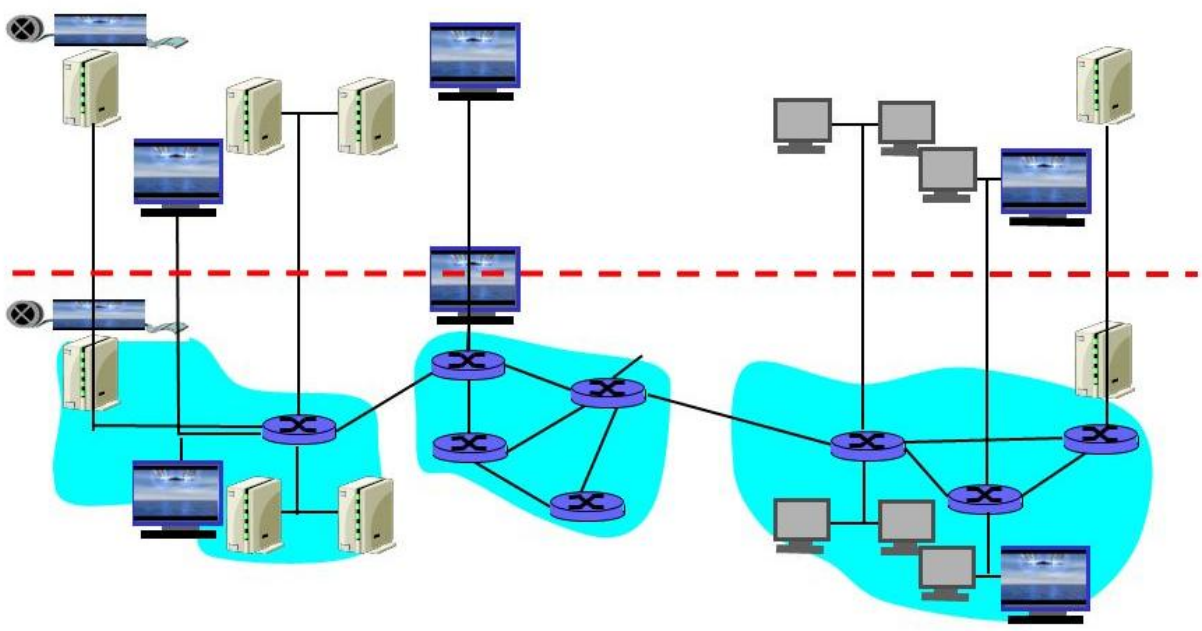

Fig 1: Centralized directory P2P network architecture

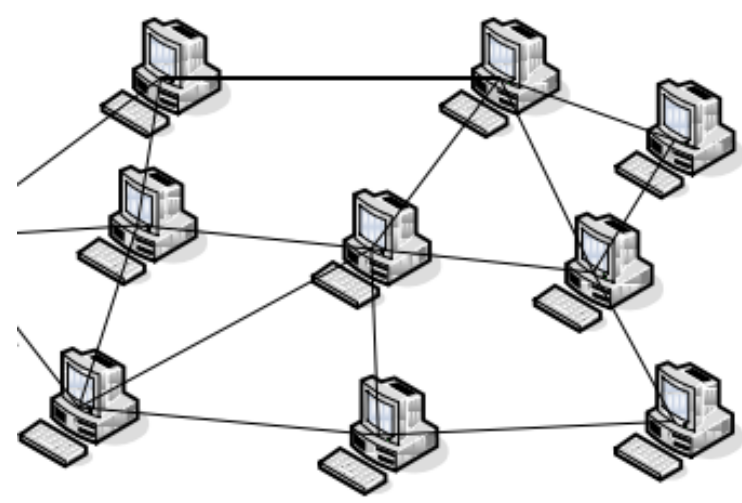

Fig 2:Pure P2P network architecture

\section{Introduction of SIP protocol}

In the Internet, there are many applications that need to create and manage sessions, and these session participants may be mobile, they may be addresses set by multiple names, or they may be contacted by different media. Many related protocols are generated, which support different real-time media sessions, such as audio, video or text messages. SIP enables Internet endpoints (known as user agents) to discover each other and negotiate their common session descriptions to work with these protocols. SIP is an application layer control protocol that can establish, modify and terminate multimedia sessions such as Internet phone calls. It is independent of the underlying protocol. The transport layer can use UDP (User Datagram Protocol) or TCP (transmission control

ISSN: 0010-8189 
protocol).

SIP itself is not an integrated communication system. SIP is designed as a part of the whole IETF multimedia data and control structure. It works with other IETF protocols to build a complete multimedia service delivery system. Typically, this architecture includes RSVP (Resource Reservation Protocol), RTP (real time Transport Protocol), RTSP (real time streaming protocol) and SDP (Session Description Protocol). SIP protocol should cooperate with these protocols to provide users with complete services. But the function and operation of SIP do not depend on any of the above protocols. It should be noted that SIP protocol itself does not provide services or services, but sip provides basic primitives for different services.

\subsection{SIP functional entity}

There are different functional entities in SIP network to complete the corresponding functions. There are mainly two types of functional entities: user agent and server. The user agent is the terminal system element of the call, and the server is the network equipment that processes the call related signaling, mainly including the proxy server, the redirection server, the registration server and the location server. These functional entities are a logical concept. In physical distribution, several entities may be integrated in the same device.

1.User agent. User agent is a functional entity that directly interacts with users. It can represent all requests and responses of users.

2.Register the server. The registration server is the server that accepts the registration request. Its purpose is to update the location database according to the contact information specified by the user in the request, so that other users can find the user. When a user registers, the registration server needs to authenticate the user. Only after the authentication is passed can the user be considered as a legitimate user in the network.

3.Proxy server. Proxy server is the entity that routes request messages to UAS and response messages to UAC, that is, to forward SIP messages. It is essentially a logical functional entity, which can work in stateful mode or stateless mode.

4. Redirect the server. The redirection server accepts the SIP request, maps the IP address of the called party to zero (if no known address) or more new addresses, and returns them to the UAS of the client. The redirection server does not send any SIP requests, which is essentially different from the proxy server.

5. Location server. Location server stores users' location information, and its main function is to provide location query service.

\subsection{SIP operation}

1. User registration. Users must register with the network before starting or logging in to become legal network users. Registration is accomplished by sending a register request to the registration server.

2. SIP call. Taking the simplest direct call mode as an example, it is also the basis of other call modes. The direct call mode is shown in Figure 3.Both ua1 and UA2 are connected to the Internet. When ua1 wants to initiate a call, it first sends an inv ite request. After receiving the invite request from UA2, the called can accept, redirect or reject the call, but it must return a response message. If receiving the request and sending a $2000 \mathrm{~K}$ response (some temporary responses may be sent before this, such as 100trying and 180ringing), UA 1 will send an ACK after receiving $2000 \mathrm{~K}$, and then start the media streaming process. At the end of the session, UA2 sends a B Ye request, and the other party responds with $200 \mathrm{OK}$.

In addition to these two basic operations, there are proxy call and SLP redirection. 
Sync Mode

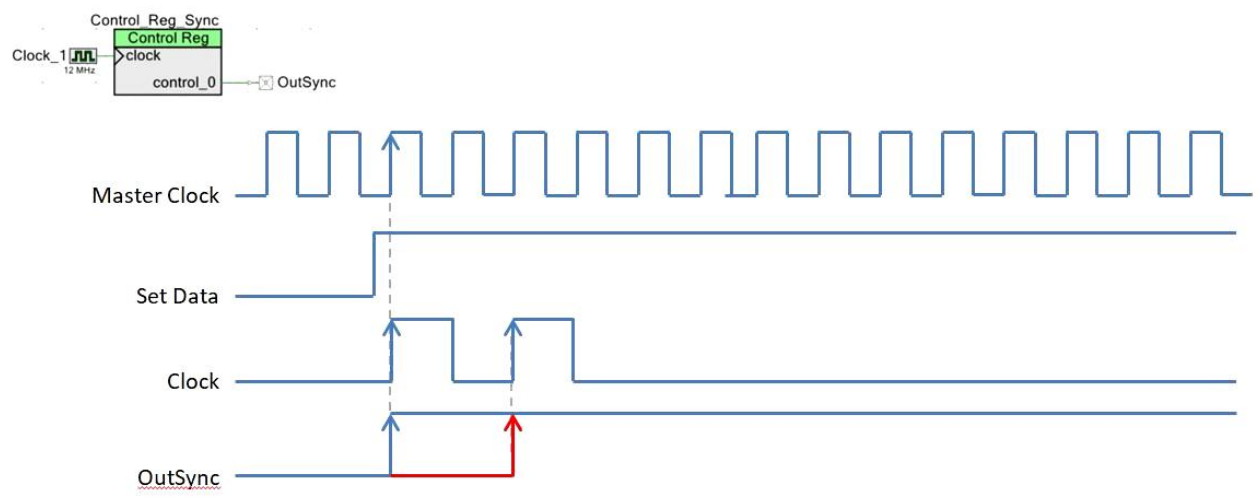

Fig 3:Direct call mode

\section{P2PSIP system design}

\subsection{P2PSIP system framework}

P2PSIP terminal prototype system is located on common nodes, and its framework is shown in fig. 4, which is mainly composed of three modules, namely user interface module, RTC (Real Time Communication) module and JXTA network module. The user interface module provides the user with an operation interface (realized by Windows programming), and calls RTC module to access JXTA network and initiate call request. RTC module and JXTA network module are connected by socket (including writing string method write (), reading string method readLine () and sending string method send (), etc.). RTC module provides S IP signaling for connection and interface method for voice and video communication. JXTA network module provides interface methods such as advertisement publishing and user positioning. Peer is a node in the network, which realizes discovery service, parsing service, aggregation service and routing service.

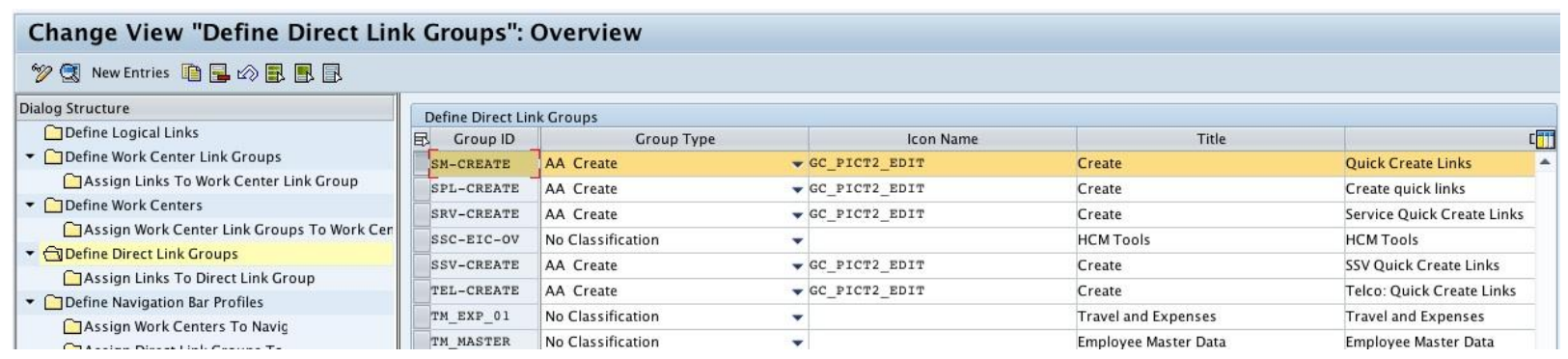

Fig 4: Frame construction

\subsection{JXTA routing mechanism}

1 .SRDI JXTA uses the distributed algorithm of SRDI to create and maintain an overall index of resources in the network. In JXTA, resources are described by metadata in the form of advertisements, and through a set of specific attributes (advertisement types, attributes and attribute values, etc.), SRDI is used to index these advertisements in the network scope. The maintained SRDI is similar to a hash table, with indexed attributes as hash keys, and the hash values are mapped to the source nodes containing the actual advertisements. Therefore, queries can be made based on these attributes anywhere on the aggregation network. In this way, SRDI can respond to the advertisement query in the network by locating the node with the required advertisement.

2. RPV and limited range traversal. A sink node must save a part of other node information to form a local sink 
node view list, which is called RPV as the known condition of route location, and must meet the consistency between RPV and global sink network view to ensure the accuracy of location. RPV is maintained by each sink node in the sink network, and it is a known list of sink nodes, which is sorted according to the unique ID of nodes. The hash function used in DHT algorithm is the same at every node, which is used to determine which sink node a query request should be forwarded to.

3. Loose and consistent DHT. In JXTA network, the convergence network layer forms a loose and consistent DHT, which is essentially a distributed data structure, including the indexes of all published advertisements in the whole JXTA network. Because of this loose and consistent DHT, distributed queries can be resolved. JXTA network operation mainly depends on this ability to resolve distributed queries.

\section{Implementation of P2PSIP system}

\subsection{RTC module}

RTC Client API is a set of API for instant messaging proposed by Microsoft corporation. RTC uses standard protocols for communication, such as SIP, RTP, etc., with good interoperability. According to this system, the basic code model of RTC is COMCComponent ObjectModel, and the main classes include RTCMain, Session class, voice session class aVISession and video session class AVSession. Fig. 5 describes the RTC module class structure and its relationship. RTCMain is the main class, which implements user login DoLogon (), start session DoCall (), CreateSession createsession (), user logout DoLogoff (), and register window RegisterClass () and window procedure windowProc (). IMSession and AVSession are inherited from Session. Class Session encapsulates the session-related process, but it is more of an abstract interface of a session, and the more concrete implementation lies in class IMSession and class AVSession.

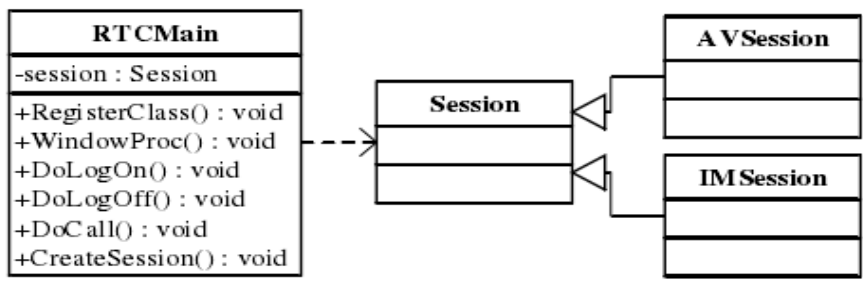

Fig 5:RTC module class structure and its relationship

5.2 JXTA network construction

Fig. 6 is a time sequence flow chart of initializing JXTA network. It can be seen from the figure that the initialization of JXTA network needs two steps: building a peer platform and building a peer group.

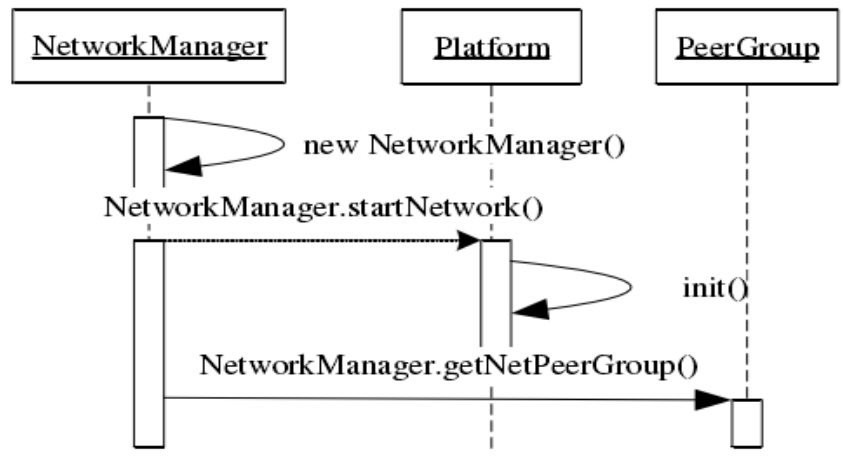

Fig 6:Sequence flow of JXTA network initialization

ISSN: 0010-8189 


\subsection{Peer join}

Before connecting with the sink node, it is necessary to discover the sink node by discovering the sink node advertisement. Once a sink node is discovered, Peer can use endpoint service to send a request to the sink node, requesting to use "JXTA propagate" as the service name and "PeerGroupID" as the service parameter. As a sink node, it is necessary to publish its own advertisement information to the network by publishing the advertisement of the sink node.

\subsection{User registration}

User UA REGISTERs with Peer node by calling DoLogon () method, that is, sending SIP REGISTER request message. After receiving the register message, Peer sends $200 \mathrm{OK}$ to UA, and the registration is successful.

\subsection{Session establishment}

The Main class main implements the method startNetwork () to start JXTA network. The Publishing class publish and the Response class response both implement the $\mathrm{j}$ ava.util.Runnable interface class, that is, after the node runs, two threads are created to publish and discover resources respectively. The Response class response implements the DiscoveryListener interface class discoverylistener, which is used to monitor whether the called user is found. when the called user is found, the discoveryEvent method DiscoveryEvent () will be triggered. as the parameter of the discoveryEvent () method, the discovery event class DiscoveryResponseMsg class contains the details of the response. In the discoveryEvent () method, the socket is called to send a message to inform the caller. After receiving this message, the caller calls the CreateSession method createSession () to send an INVITE message to the called user.

\section{Conclusion}

Based on the analysis of P2P network architecture, this paper deeply studies the structure P2P network architecture based on DHT. The feasibility of the system is analyzed, including the combination of P2P and sip, the network structure and the framework of the system. Then, the JXTA routing mechanism is deeply studied, including SRDI, RPV, limited range traversal and loosely consistent DHT. In view of its shortcomings, the chord ring of consistent hash algorithm is introduced in the aggregation network layer, which can improve the query efficiency of JXTA network and the ability to adapt to the dynamic network. Finally, a prototype system of p2p sip based on JXTA is designed and implemented, including user interface module, RTC module and JXTA network module. JXTA network module includes discovery service, resolution service, aggregation service and endpoint routing service. Finally, the feasibility of the system is verified by function test.

\section{References}

[1] Jiang Wei, Fang Binxing, Tian Zhihong. Network Security Evaluation and Optimal Active Defense Based on Attack Defense Game Model. Acta Computer Sinica, 2009, 32 (004): 817-827

[2] Miao Yongqing. Stochastic Model Method and Evaluation Technology of Network Security. China Science and Technology Investment, 2017, 4: 314

[3] Yi Hua Zhou, Wei Min Shi, Wei Ma. Research on Computer Network Security Teaching Mode for Postgraduates Under the Background of New Engineering. Innovation and Practice of Teaching Methods, 2020, 3 (14): 169

[4] Li Weiming, Lei Jie, Dong Jing. an Optimized Real-time Network Security Risk Quantification Method. Acta Computa Sinica, 2009 (04): 793-804

[5] Bao Xiuguo, Hu Mingzeng, Zhang Hongli. Two Quantitative Analysis Methods for Survivability of Network Security Management Systems. Acta Communication Sinica, 2004, 25 (9): 34-41

[6] Yang Yi, Bian Yuan, Zhang Tianqiao. Network Security Situation Awareness Based on Machine Learning. Computer Science and Application, 2020, 10 (12): 8

[7] Li Zhiyong. Hierarchical Network Security Threat Situation Quantitative Assessment Method. Communication World, 2016, 23: 70-70 
[8] Hu Wenji, Xu Mingwei. Analysis of Secure Routing Protocols for Wireless Sensor Networks. Journal of Beijing University of Posts and Telecommunications, 2006, 29 (s1): 107-111

[9] Wei Yonglian, Yi Feng, Feng Dengguo, Yong W, Yifeng L. Network Security Situation Assessment Model Based on Information Fusion. Computer Research and Development, 2009, 46 (3): 353-362

[10] Xu Guoguang, Li Tao, Wang Yifeng. A Network Security Real-time Risk Detection Method Based on Artificial Immune. Computer Engineering, 2005,31 (12): 945-949 